\title{
Reprodutibilidade e Extensibilidade de Datasets de Rede: um estudo da replicação de traces de pacotes.
}

\author{
Luciano B. Fiorino, Maxwell E. Monteiro, \\ Cristina K. Dominicini e Gilmar L. Vassoler \\ Programa de Pós-graduação em Computação Aplicada (PPComp) \\ Campus Serra do Instituto Federal do Espírito Santo (IFES) \\ Serra, Brasil \\ \{lbiancardi,maxmonte,cristina.dominicini,gilmarvassoler\}@ifes.edu.br
}

\author{
João H. Corrêa e \\ Rodolfo S. Villaça \\ Programa de Pós-Graduação em Informática (PPGI) \\ Universidade Federal do Espírito Santo (UFES) \\ Vitória, Brasil \\ jhenrique@gmail.com,rodolfo.villaca@ufes.br
}

\begin{abstract}
Resumo-Em geral, a aplicação de algoritmos de aprendizado de máquina em problemas de redes utilizam datasets gerados a partir de traces de pacotes. Entretanto, o processo de geração dos datasets atuais não segue critérios que permitam identificar as informações necessárias para a reprodução e extensão dos mesmos. Dessa forma, este trabalho realiza um estudo detalhado sobre formas e ferramentas para reprodução dos tráfegos de rede dos datasets. Diante dos problemas de reprodutibilidade identificados, propomos uma metodologia para geração de datasets de traces de pacotes, de forma a minimizar esses problemas, possibilitando a reprodução dos seus tráfegos de rede e estendêlos com novos dados.
\end{abstract}

Index Terms-replicação de tráfego, traces de pacotes, computação em nuvem, datasets, aprendizado de máquina.

\section{INTRODUÇÃO}

Nos últimos anos, técnicas de aprendizado de máquina têm sido muito utilizadas para solução de problemas complexos em redes, como previsão de tráfego, roteamento, classificação, controle de congestionamento, gerenciamento de recursos e segurança de rede [1]. Em geral, o aprendizado de máquina é aplicado para encontrar padrões ou tendências em um conjunto grande e representativo de dados.

Entretanto, o desenvolvimento de soluções que aplicam as técnicas de aprendizado de máquina à área de redes sofre com problemas de precisão na sua avaliação, comparação e implantação, devido à escassez de conjuntos de dados adequados [2]. Isso acontece porque não se conhece uma metodologia que forneça critérios para descrever o ambiente, planejar e gerar o tráfego, coletar métricas e finalmente gerar as amostras dos datasets, tornando difícil a comparação de diferentes conjuntos de dados e avaliar sua adequação para diferentes problemas de rede [1].

Em particular, o processo de criação de datasets na área de redes, tipicamente, utiliza métricas referentes aos fluxos de rede, obtidas, a exemplo, da análise de traces de pacotes em arquivos do tipo PCAP $[3]$.

No entanto, ao analisar os datasets disponibilizados na literatura, observa-se a falta de informações importantes no processo de criação, tais como: o cenário do experimento detalhado, os softwares e métodos utilizados na geração dos

\footnotetext{
${ }^{1}$ https://www.tcpdump.org/
}

tráfegos de rede. A falta de informações torna difícil, e por vezes impossível, a reprodução dos traces de pacotes para verificação de resultados ou coleta de novas métricas, que permitiriam estender o conjunto de características do dataset. Como consequência, quando novas pesquisas necessitam de métricas não presentes nos datasets existentes, é necessário gerar suas próprias bases de dados para os experimentos, pois existem dificuldades em reproduzir datasets existentes.

Em se tratando de algoritmos de aprendizado de máquina, gerar a própria base de dados requer muito cuidado para que existam dados representativos para todos os casos, de modo a evitar erros de generalização nos modelos de aprendizado [4]. Assim, a reprodução de datasets confiáveis, gerando novas bases e/ou estendendo os mesmos com novas métricas, pode reduzir o risco de modelos de aprendizado tendenciosos. A reprodução de datasets também pode contribuir para comparações entre técnicas de aprendizado de máquina, além da aplicação dos tráfegos gerados originalmente em novos ambientes de interesse.

Para abordar o problema de reprodutibilidade e extensibilidade dos datasets de redes existentes, este trabalho propõe uma metodologia para geração de datasets de traces de pacotes, com critérios mínimos e um conjunto de etapas, que permite a reprodução e extensão dos mesmos em novas pesquisas. $\mathrm{O}$ ponto de partida para elaborar a metodologia será a avaliação de datasets disponíveis na literatura e de ferramentas abertas para replicação de traces de pacotes, com o objetivo de analisar a reprodutibilidade dos datasets avaliados para coletar novas métricas.

Como principais contribuições deste trabalho, pode-se destacar: (i) identificação das deficiências dos datasets em termos de reprodutibilidade e extensibilidade; (ii) identificação das deficiências das ferramentas de replicação de tráfegos de rede capturados em arquivos do tipo PCAP; (iii) proposta de metodologia com critérios e melhores práticas para geração de um dataset com informações suficientes para garantir sua reprodutibilidade e extensibilidade; e (iv) avaliação da replicação de traces de pacotes contidos em datasets da literatura com ferramentas de replicação abertas, de modo a coletar novas métricas de interesse, gerando novos conjuntos de dados e/ou estendendo os atuais. 
Para avaliar a replicação traces de pacotes, este artigo apresenta um experimento baseado na arquitetura de nuvem OpenStack [5]. Este cenário foi escolhido como prova de conceito, pois as arquiteturas de nuvem possuem mecanismos nativos de monitoramento, os quais fornecem novas métricas não exploradas nos datasets de redes tradicionais (e.g., uso de CPU, consumo de memória e uso de disco). O experimento mostrou problemas de reprodutibilidade a partir da replicação de traces de pacotes, os quais enfatizam a necessidade da proposta de geração de datasets apresentada.

Este artigo está organizado da seguinte forma. A Seção 2 mostra os trabalhos relacionados. A Seção 3 apresenta a proposta de geração de datasets. A Seção 4 apresenta um estudo das capacidades das ferramentas de replicação disponíveis. A Seção 5 apresenta a avaliação da replicação de traces de pacotes. Por fim, a Seção 6 apresenta as conclusões.

\section{TRABALhOS RELACIONADOS}

Esta seção compara oito datasets da literatura em relação a um conjunto de características, importantes para garantir a reprodutibilidade e extensibilidade de datasets de redes. A Tabela I apresenta uma síntese da análise dos datasets, destacando suas características para extração dos tráfegos nos arquivos de captura e a replicação destes tráfegos.

$\mathrm{O}$ detalhamento da topologia de rede permite identificar os elementos participantes do cenário e a matriz de tráfego. Essas informações são essenciais para identificação da origem e destino dos traces de pacotes do dataset.

Arquivos PCAP são gerados a partir de capturas de traces de pacotes com ferramentas como o Tcpdump e Wireshark. Os arquivos PCAP são indispensáveis para a reprodutibilidade dos traces de pacotes de rede. Estes arquivos podem chegar a centenas de gigabytes, dependendo da duração do tráfego.

Em geral, o MTU padrão das redes é de 1500 bytes ${ }^{2}$ A existência de pacotes com tamanho maior que o MTU da rede utilizada é reflexo da captura ter sido feita com recursos de Offloads ${ }^{3}$ habilitados na interface de rede. Esses pacotes tipicamente apresentam problemas para a replicação, pois as ferramentas de replicação devem ser capazes de fragmentar os pacotes para se adequar ao MTU, caso contrário haverá descarte de pacotes e o tráfego não será replicado fielmente. É importante enfatizar que nos tráfegos capturados com Offloads habilitados, o número de pacotes no arquivo PCAP difere do número real transmitidos na rede, impactando em métricas como o número de pacotes recebidos/enviados.

Os timestamps de início e fim dos tráfegos nas amostras do dataset informam o intervalo de tempo do respectivo tráfego, para o qual a amostra foi gerada. Estas são informações essenciais para extrair com exatidão o trace de pacotes da tupla TCP/UDP em cada amostra. Idealmente, a precisão dos timestamps de início e fim dos tráfegos deve ser igual à precisão dos timestamps dos pacotes no arquivo PCAP.

${ }^{2}$ https://www.rfc-editor.org/rfc/rfc894.txt html
TABELA I

CARACTERÍsticas dos Datasets AVAliados

\begin{tabular}{|c|c|c|c|c|c|c|}
\hline Dataset & Topologia ${ }^{1}$ & $\begin{array}{l}\text { Arquivo } \\
\text { PCAP }\end{array}$ & \begin{tabular}{|l|} 
Pacotes \\
MTU \\
padrão
\end{tabular} & $\begin{array}{l}\text { Timestamp }{ }^{2} \\
\text { de início } \\
\text { e fim }\end{array}$ & \begin{tabular}{|l} 
Dados \\
de treino \\
e teste
\end{tabular} & $\begin{array}{l}\text { Dados } \\
\text { rotulados }\end{array}$ \\
\hline CIC-DDoS2019 & $\checkmark$ & $\checkmark$ & $x$ & $x$ & $\checkmark$ & $\checkmark$ \\
\hline CIC-IDS2017 [7] & $\checkmark$ & $\checkmark$ & $x$ & $x$ & $x$ & $\checkmark$ \\
\hline$C T U-13[8]$ & $x$ & $\checkmark$ & $x$ & $\sqrt{ }^{3}$ & $\checkmark$ & $\checkmark$ \\
\hline$I S C X-I D S-2012$ & $\checkmark$ & $\checkmark$ & $\checkmark$ & $x^{4}$ & $x$ & $\checkmark$ \\
\hline UNSW-NB15 9] & $\checkmark$ & $\mathfrak{S}^{5}$ & $\checkmark$ & $x$ & $x^{6}$ & $\checkmark$ \\
\hline NDSec-I [10] & $(\checkmark)$ & $\checkmark$ & $x$ & $(\checkmark)^{7}$ & $x$ & $\checkmark$ \\
\hline \begin{tabular}{ll|l} 
KDDCup'yg & $11]$
\end{tabular} & $x$ & $x$ & - & - & $\checkmark$ & $\checkmark$ \\
\hline$N S L K D D$ 12] & $x$ & $x$ & - & - & $\checkmark$ & $\checkmark$ \\
\hline \multicolumn{7}{|c|}{$\mathcal{V}=$ atende, $(\boldsymbol{V})=$ atende parcialmente, $\boldsymbol{X}=$ não atende, " $-"=$ não disponível } \\
\hline $\begin{array}{l}1 \text { Topologia e matri } \\
2 \text { Timestamp de iní } \\
3 \text { Timestamp de iníc } \\
5 \text { Linux cooked-mo } \\
6 \text { A partir de separ } \\
7 \text { Possui diferença }\end{array}$ & $\begin{array}{l}\mathrm{Z} \text { de tráfego } \\
\text { io e fim dos } \\
\text { io e duraçãc } \\
\text { le capture ( } \\
\text { ç̧ões do dad } \\
\text { le } 03 \text { casas }\end{array}$ & $\begin{array}{l}\text { detalhada; } \\
\text { tráfegos } \\
{ }^{4} \text { Não } \mathrm{c} \\
L L) \text {, não } \\
\text { os; } \\
\text { lecimais. }\end{array}$ & $\begin{array}{l}\text { nas amos } \\
\text { ontém ca } \\
\text { contém } E\end{array}$ & $\begin{array}{l}\text { tras; } \\
\text { sas decimais; } \\
\text { thernet heade }\end{array}$ & & \\
\hline
\end{tabular}

Dados de treino e teste são as partes do dataset utilizadas para o treinamento e, posteriormente, o teste dos algoritmos de classificação. Um dataset que não tenha dados distintos de treino e teste, necessita ser dividido manualmente, podendo gerar problemas no modelo devido a falta de aleatoriedade nos conjuntos de dados. Dessa forma o conjunto de treino pode conter dados não presentes no conjunto de teste e vice-versa, impactando no desempenho dos modelos de aprendizado [4].

Ter os dados rotulados significa que cada amostra do dataset foi classificada para cada tipo de tráfego, uma característica fundamental para o treinamento dos algoritmos de classificação. O rótulo possibilita filtrar as amostras de cada tipo de tráfego e juntamente com as características anteriores, extrair dos arquivos PCAP os respectivos tráfegos de interesse.

\section{Proposta de Geração de Datasets REPRODUZÍVEIS E EXTENSÍVEIS}

Esta seção apresenta uma proposta para geração de datasets baseados em traces de pacotes, elaborada a partir da análise dos datasets e das ferramentas de replicação de traces de pacotes, de forma a assegurar a reprodutibilidade dos traces de pacotes. A Fig. 1 ilustra as etapas da metodologia.

1. Elaboração do Cenário: Alguns datasets não detalham a topologia de rede, matriz de tráfego, MTU e a taxa de transferência da rede. Dessa forma não é possível, em alguns casos, identificar com precisão os tráfegos a partir dos arquivos de traces de pacotes, o que inviabiliza a reprodução do cenário original. É necessário que a topologia e a matriz de tráfego sejam detalhadas para identificação dos hosts e reprodução dos tráfegos. A informação do $\boldsymbol{M T U}$ da rede é importante para reprodução do cenário, uma vez que as redes podem ter diferentes valores de MTU dependendo do protocolo de encapsulamento utilizado. A taxa de transferência da rede é necessária para evitar o problema de reproduzir tráfegos em redes de menor capacidade, o que gera padrões diferentes para estes mesmos tráfegos. Outro ponto muito importante são os hosts participantes do cenário, em especial os de destino dos fluxos. Muitos datasets não fornecem informações dos servidores alvos dos tráfegos. Há casos, como no trabalho [13], em que é utilizado um ambiente privado, gerando problemas legais 

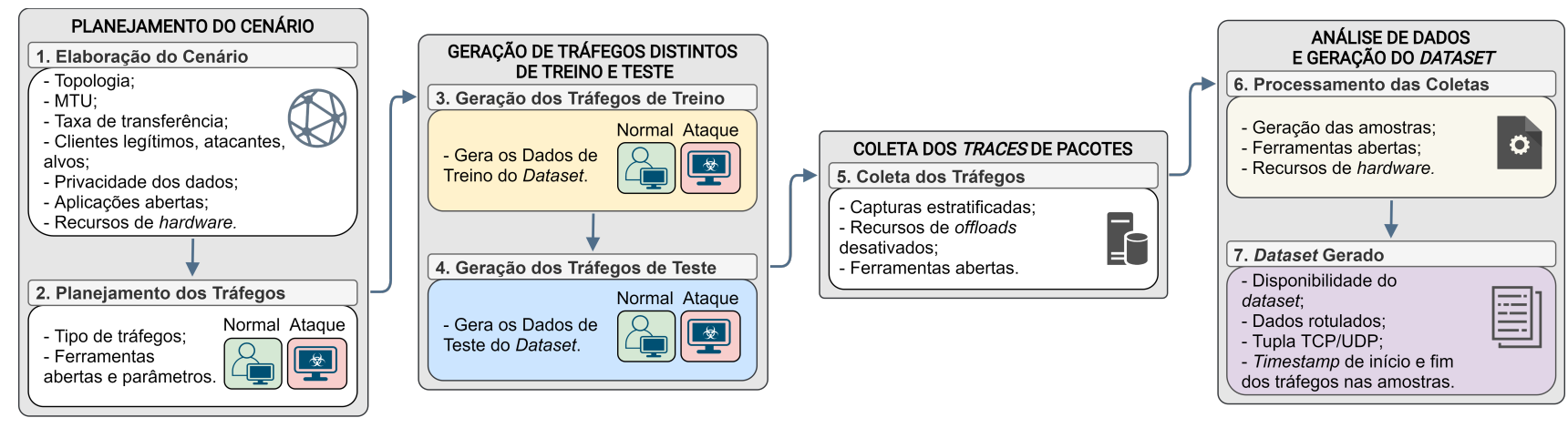

Fig. 1. Proposta de geração de Dataset.

quanto a privacidade dos dados trafegados e sua disponibilidade. A indisponibilidade das aplicações hospedadas inviabiliza a reprodução do cenário original e a replicação dos tráfegos. Para solucionar estes problemas, é recomendado o uso de aplicações abertas e dados anonimizados/fictícios em um cenário de teste para eliminar o problema de privacidade e permitir a reprodução do servidor alvo. As informações de hardware possibilitam reproduzir fielmente os servidores, o que permite comparar as métricas originalmente coletadas com outras não exploradas para um tráfego equivalente reproduzido e com a mesma carga de processamento.

2. Planejamento dos Tráfegos: Observa-se nos datasets pouco detalhamento nos métodos utilizados na geração dos tráfegos, onde muitas vezes apenas a ferramenta utilizada é citada. Detalhar a forma de geração dos tráfegos, com informações do tipo do tráfego, da duração, do número de clientes e/ou atacantes do cenário, bem como das ferramentas e seus parâmetros de configuração, permitem reproduzir um tráfego equivalente ao original, uma vez que não há ferramentas abertas capazes de replicar tráfegos capturados em modo stateful igual ao original. Dessa forma, encoraja-se que as ferramentas e os parâmetros de configuração utilizados para geração de tráfego devem ser abertos.

3. Tráfegos de Treino / 4. Tráfegos de Teste: De acordo com [4], a melhor forma de testar um modelo de aprendizado de máquina é a separação dos dados em conjuntos de treino e teste, de forma aleatória para evitar problemas na classificação devido à falta ou existência de poucas amostras de determinada classe no conjunto de treinamento. Ter tráfegos normal e de ataque distintos para geração de cada conjunto de treino e teste elimina este problema, pois haverá amostras representando todos os tipos de tráfegos em ambos os conjuntos.

5. Coleta dos Tráfegos: Os datasets da literatura são gerados após análise e processamento dos arquivos de traces de pacotes e, normalmente, disponibilizados como um único arquivo do tipo PCAP. Nos testes realizados, observou-se que um arquivo de captura com alguns gigabytes requer alto poder computacional para processar a separação de tráfegos, devido à grande quantidade de pacotes que precisam ser analisados. Uma solução para esse problema é a captura de forma estratificada, onde, para cada tráfego, um arquivo de captura é gerado. Outro ponto está relacionado com os recursos de Offloads, que devem estar desativados na interface de rede para que o arquivo de captura contenha os pacotes com tamanho dentro do MTU da rede utilizada no experimento, tipicamente no padrão de 1500 bytes. Também foi observado que algumas ferramentas, como em [14] e [15], não utilizam o formato aberto PCAP para os traces de pacotes, dessa forma é recomendada a utilização de ferramentas e padrões abertos para permitir a reprodução do experimento original.

6. Processamento das Coletas: Para ser possível reproduzir as amostras de um dataset, é necessário que o método de geração dessas amostras seja detalhado e as métricas de rede (características) as quais compõem o dataset sejam identificadas. Além do método de geração, os seguintes pontos devem ser considerados: as ferramentas de análise, que devem estar disponíveis para utilização; e os recursos de hardware que foram necessários para processamento, uma vez que processar grandes arquivos PCAP requer maior poder computacional.

7. Dataset Gerado: O conjunto de dados gerado deve estar disponível, e além de ter seus dados devidamente rotulados, deve conter: i) conjuntos distintos de treino e teste para avaliação dos modelos de aprendizado de máquina, conjuntos estes gerados a partir dos tráfegos de treino e teste; ii) a tupla TCP/UDP nas amostras, facilitando a identificação da matriz de tráfego; iii) os timestamps de início de fim do tráfego nas amostras, com a mesma precisão dos pacotes capturados, para se extrair com exatidão os traces de pacotes referentes às amostras nos arquivos de captura.

A hipótese deste trabalho é que datasets gerados levando em consideração as informações descritas nas etapas acima, facilitariam a reprodutibilidade de seus traces de pacotes, consequentemente permitindo a extensão dos datasets por meio de coletas de novas características.

\section{Estudo das CApacidades das FerRamentas de REPLICAÇÃO DisPonívEIS}

Esta seção avalia doze ferramentas de código aberto, identificadas na literatura, capazes de replicar tráfegos de rede a partir de trace de pacotes capturados. A Tabela II sumariza a avaliação realizada, considerando as seguintes características: modo de tráfego, suporte a arquivos PCAP, suporte a pacotes maiores que o MTU padrão, replicação com preservação do 


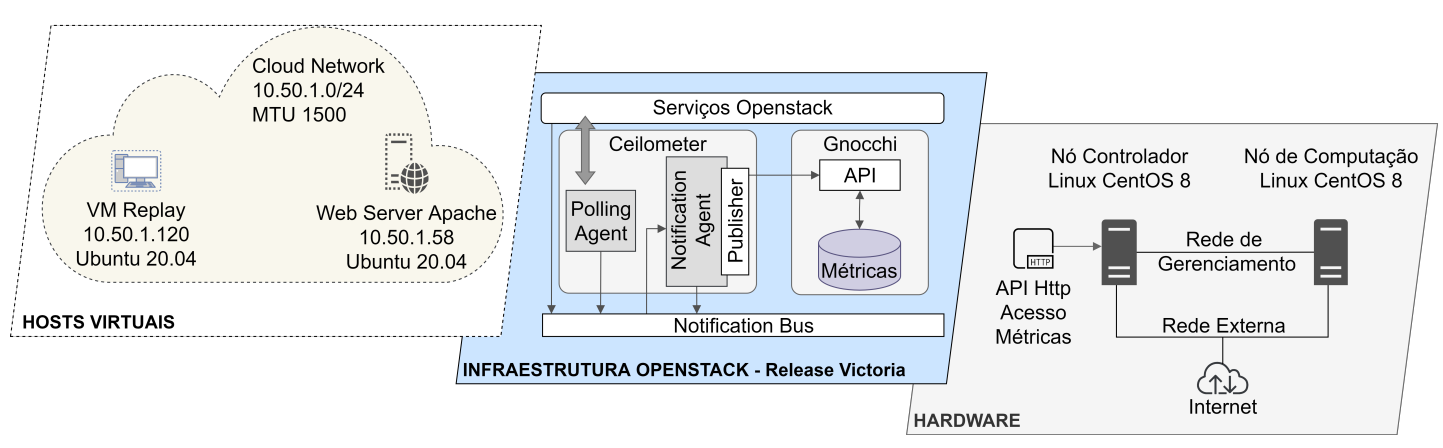

Fig. 2. Cenário do experimento.

$\mathrm{O}$ experimento teve como premissa preservar os timestamps dos pacotes durante a replicação, de modo a enviar os pacotes nos mesmos intervalos de tempo, tornando o tráfego replicado o mais próximo do original. Para isso foram escolhidas as ferramentas Tcpreplay, Moongen, GopherCap e GoReplay.

Para fins de comprovação, os códigos utilizados, os arquivos PCAP com os tráfegos de rede selecionados e preparados para replicação, e o dataset gerado no experimento deste trabalho estão disponíveis em: https://lbfiorino.github.io/

\section{B. Novas Métricas para os Tráfegos de Interesse do Dataset}

Como dados de interesse para o experimento, foram escolhidas métricas que variam de acordo com a carga de utilização das instâncias da nuvem em função da replicação do tráfego. Ao todo, foram selecionadas doze métricas relacionadas às instâncias, sendo elas: cpu usage, memory usage, memory swap in, memory swap out, disk device read requests, disk device write requests, disk device read bytes, disk device write bytes, network incoming bytes, network outgoing bytes, network incoming packets, network outgoing packets.

A coleta dos dados foi realizada por uma aplicação Python através da API do serviço Gnocchi e de forma offline, após a replicação dos tráfegos. Para isso, os relógios dos hosts foram sincronizados para a coleta ser realizada no intervalo de tempo entre o início e o fim da replicação. A nuvem utilizada gerava as métricas a cada 5 segundos. Assim, cada amostra do dataset gerado corresponde a coletas das métricas a cada 5 segundos.

\section{Seleção e Preparação dos Tráfegos para Replicação}

Dentre os datasets avaliados, o NDSec-1 [10] foi escolhido por disponibilizar as seguintes informações, as quais permitem extrair tráfegos de interesse do arquivo PCAP satisfatoriamente: i) detalhes da topologia de rede; ii) amostras rotuladas em tráfego normal e de ataque; iii) tráfego normal e de ataque para um mesmo destino, identificado com análise da tupla TCP nas amostras; iv) intervalos de tempo dos tráfegos nas amostras; v) arquivos PCAP menores, que reduziram o tempo de processamento. Apesar de não disponibilizada, foi identificado nos traces o uso da aplicação web aberta DVWA7] possibilitando a reprodução do servidor web.

\footnotetext{
7 https://dvwa.co.uk/
}

$\mathrm{O}$ vetor de ataque SYN-Flood é um dos dominantes na Internet [26]. Diante disso, foram escolhidos os tráfegos de ataque SYN-Flood e o tráfego normal HTTP do dataset, ambos para o mesmo destino. A escolha do cenário de tráfegos contidos no dataset NDSec-1 foi baseada nos seguintes fatores:

1) Menor custo computacional para processamento do arquivo PCAP. Para cada amostra do dataset, é necessário um processo para extrair do arquivo PCAP os pacotes do fluxo referente à amostra, pois a informação de início e fim do fluxo está na amostra. Para isso, foi utilizada a ferramenta Tshark ${ }^{8}$, que não suporta multithreading. A diferença de três casas decimais na precisão do timestamp entre as amostras e os pacotes capturados agrava o custo computacional devido a passos adicionais no processo de extração, que foi possível combinando a informação do timestamp com o número do frame do pacote no arquivo PCAP. Por estes motivos, foi escolhido o arquivo PCAP do cenário botnet do dataset.

2) Pacotes adequados ao MTU padrão. Após análise dos traces de pacotes do cenário botnet, foram encontrados tráfegos de ataque SYN-Flood e tráfego normal HTTP adequados ao MTU padrão, os quais puderam ser utilizados no experimento.

Uma vez escolhidos os tráfegos, os mesmos foram extraídos do arquivo PCAP original, gerando um arquivo PCAP para cada tráfego de forma estratificada. A extração dos pacotes de cada tráfego foi feita de acordo com a tupla TCP, o timestamp de início e fim, e o rótulo contido em cada amostra.

Por fim, os pacotes extraídos precisaram ser modificados para se adequar à arquitetura de replicação. As seguintes alterações foram realizadas nos pacotes: (i) alteração da tupla TCP (endereço MAC e IP de origem e destino) para ambos os tráfegos, normal e ataque; e (ii) no tráfego normal, os campos host e referer do protocolo HTTP foram alterados para o host do servidor web do novo cenário. Por fim, os checksums IP/TCP foram recalculados. Para este processo foram utilizadas as ferramentas scapy ${ }^{9}$ e tcprewrite [16].

\section{Replicação dos tráfegos}

Os tráfegos de interesse foram replicados em momentos diferentes, portanto, sem concorrência dos tráfegos. As cap-

${ }^{8}$ https://www.wireshark.org/ 
turas dos tráfegos replicados foram feitas no host Nó de Computação apresentado na Fig. 2

A ferramenta GoReplay foi utilizada para replicar o tráfego stateful HTTP. As requisições foram extraídas do arquivo PCAP e salvas em um novo arquivo, no padrão da ferramenta. A replicação foi realizada de forma esperada, com duração de $0,014 \%$ superior ao original. Enfatiza-se que o tráfego replicado não envolvia processos de autenticação sofisticada.

O tráfego SYN-Flood foi replicado utilizando as ferramentas Tcpreplay, MoonGen e GopherCap, devido a este tipo de ataque não requerer o estabelecimento da conexão, sendo necessário apenas o envio do pacote SYN ao host de destino. As ferramentas apresentaram bons resultados, onde os tráfegos replicados tiveram durações superiores ao original de $0,013 \%$, $0,351 \%$ e $0,672 \%$ respectivamente.

Apesar dos tráfegos selecionados terem sido replicados com sucesso, o volume pequeno de tráfego não alterou consideravelmente as métricas escolhidas. Dessa forma, é necessário tráfegos massivos para possibilitar uma análise comparativa entre os datasets nos algoritmos de aprendizado de máquina.

Importante notar que o experimento demonstrou a possibilidade de replicação de tráfegos específicos, tais como SYNFlood e HTTP simples. No entanto, há carência de ferramentas abertas para replicação satisfatória de outros tipos de tráfegos, bem como a necessidade de uma metodologia para gerar novos datasets, com as informações para facilitar a extração e reprodução dos fluxos de interesses.

\section{CONCLUSÃO}

Este trabalho analisou as deficiências, tanto nos datasets da literatura quanto nas ferramentas de replicação de traces de pacotes, para gerar novas bases de dados e/ou estender as atuais por meio de coleta de métricas de nuvem. A avaliação identificou problemas desde a falta de informações a respeito dos experimentos dos datasets quanto deficiências das ferramentas abertas para replicação de tráfego stateful. A indisponibilidade das ferramentas e parâmetros utilizados na geração dos tráfegos originais gera a necessidade de novas ferramentas de replicação. Diante dessas limitações, concluise que não é possível reproduzir e estender satisfatoriamente os datasets de redes analisados, mas que a metodologia proposta avança em identificar os passos para permitir que novos datasets sejam gerados para preencher esta lacuna.

Como trabalhos futuros, pretendemos gerar um dataset de rede próprio, utilizando a metodologia proposta, com tráfegos massivos, os quais possam ser replicados ou reproduzidos com as ferramentas atuais de acordo com suas especificidades. Isso permitirá realizar o ciclo completo da metodologia para gerar o dataset, reproduzi-lo e estendê-lo com novas métricas. Por fim, esperamos avaliar a relevância de novas métricas na solução de problemas usando técnicas de aprendizado de máquina.

\section{AGRADECIMENTOS}

Agradecemos ao IFES pelo apoio financeiro via Programa Institucional de Apoio à Pós-graduação Stricto Sensu (Propós) e à FAPES pelo suporte ao laboratório CIDIG do Centro de Pesquisa, Inovação e Desenvolvimento do Espírito Santo.

\section{REFERÊNCIAS}

[1] R. Boutaba et al., "A comprehensive survey on machine learning for networking: evolution, applications and research opportunities," Journal of Internet Services and Applications, vol. 9, no. 1, p. 16, 2018.

[2] A. Shiravi et al., "Toward developing a systematic approach to generate benchmark datasets for intrusion detection," Computers \& Security, vol. 31, no. 3, pp. 357-374, 2012.

[3] M. A. Ferrag et al., "Deep learning for cyber security intrusion detection: Approaches, datasets, and comparative study," Journal of Information Security and Applications, vol. 50, p. 102419, 2020.

[4] A. Géron, Hands-on machine learning with Scikit-Learn, Keras, and TensorFlow: Concepts, tools, and techniques to build intelligent systems. O'Reilly Media, 2019, ch. 1, pp. 24-31.

[5] O. I. F. OpenStack, "Openstack - open source cloud computing platform software," https://www.openstack.org/software// acesso em: 25 fev. 2021.

[6] I. Sharafaldin et al., "Developing realistic distributed denial of service (ddos) attack dataset and taxonomy," in ICCST, 2019, pp. 1-8.

[7] — " "Toward generating a new intrusion detection dataset and intrusion traffic characterization," in ICISSP, 2018, pp. 108-116.

[8] S. García et al., "An empirical comparison of botnet detection methods," Computers \& Security, vol. 45, pp. 100-123, 2014.

[9] N. Moustafa and J. Slay, "Unsw-nb15: a comprehensive data set for network intrusion detection systems (unsw-nb15 network data set)," in MilCIS, 2015, pp. 1-6.

[10] F. Beer et al., "A new attack composition for network security," in 10. DFN-Forum Kommunikationstechnologien, P. Müller, B. Neumair, H. Raiser, and G. Dreo Rodosek, Eds. Bonn: Gesellschaft für Informatik e.V., 2017, pp. 11-20.

[11] I. University of California, "Kdd cup 1999 data," http://kdd.ics.uci.edu/ databases/kddcup99/kddcup99.html 1999, acesso em: 20 fev. 2021.

[12] M. Tavallaee et al., "A detailed analysis of the kdd cup 99 data set," in 2009 IEEE Symposium on Computational Intelligence for Security and Defense Applications, 2009.

[13] F. Beer and U. Bühler, "Feature selection for flow-based intrusion detection using rough set theory," in 2017 IEEE 14th ICNSC, May 2017 , pp. 617-624.

[14] W.-c. Feng et al., "Tcpivo: A high-performance packet replay engine," in Proceedings of the ACM SIGCOMM Workshop on Models, Methods and Tools for Reproducible Network Research. New York, NY, USA: ACM, 2003, p. 57-64.

[15] Y. Li, R. Miao, M. Alizadeh, and M. Yu, "DETER: Deterministic TCP replay for performance diagnosis," in 16th USENIX Symposium on Networked Systems Design and Implementation (NSDI 19). Boston, MA: USENIX Association, feb 2019, pp. 437-452.

[16] AppNeta, "Tcpreplay - pcap editing and replaying utilities," https: //tcpreplay.appneta.com/ 2020, acesso em: 20 jan. 2021.

[17] Stamus Networks, "Gophercap: Accurate, modular, scalable pcap manipulation tool written in go." https://github.com/StamusNetworks/ gophercap 2020, acesso em: 08 fev. 2021.

[18] P. Emmerich et al., "Moongen: A scriptable high-speed packet generator," Proceedings of the 2015 Internet Measurement Conference, Oct 2015.

[19] K. Wiles, "Pktgen - traffic generator powered by dpdk," https://github. com/pktgen/Pktgen-DPDK 2020, acesso em: 01 fev. 2021.

[20] J. Ribas, "Dpdk burst replay tool," https://github.com/FraudBuster/ dpdk-burst-replay 2019, acesso em: 01 fev. 2021.

[21] Leonid Bugaev, "Goreplay," https://goreplay.org/ 2020, acesso em: 26 mai. 2021.

[22] Y.-D. Lin et al., "Low-storage capture and loss recovery selective replay of real flows," IEEE Communications Magazine, vol. 50, no. 4, pp. 114$121,2012$.

[23] S.-S. Hong and S. F. Wu, "On interactive internet traffic replay," in International Workshop on Recent Advances in Intrusion Detection. Springer, 2005, pp. 247-264.

[24] Cisco, "Cisco t-rex," https://trex-tgn.cisco.com/ 2021, acesso em: 11 jun. 2021.

[25] J. H. Corrêa et al., "Ml-based ddos detection and identification using native cloud telemetry macroscopic monitoring," Journal of Network and Systems Management, vol. 29, no. 2, pp. 1-28, 2021.

[26] T. C. B. Cloudflare, "Network-layer ddos attack trends for q4 2020," https://blog.cloudflare.com/ network-layer-ddos-attack-trends-for-q4-2020/ 2021, acesso em: 22 mar. 2021. 